\title{
Electrostatic Potential Distribution for Spheroidal Surfaces in Symmetric Electrolyte Solutions
}

\author{
Jyh-Ping $\mathrm{Hsu}^{1}$ and Bo-Tau Liu \\ Department of Chemical Engineering, National Taiwan University, Taipei, Taiwan 10617, Republic of China
}

Received March 25, 1997; accepted June 2, 1997

The electrostatic potential distribution for a charged spheroidal surface immersed in a symmetric electrolyte solution is derived. Such surfaces simulate a wide class of dispersed entities. Two types of boundary condition at the solid surface are considered, constant surface potential and constant amount of surface charges; both conductive and nonconductive surfaces are examined for the latter. The present analysis extends the conventional one-dimensional treatment on simple geometries to a two-dimensional space. A perturbation method is adopted to solve the governing PoissonB oltzmann equation for the case of thin to moderately thick double layers. The classic results for planar and spherical surfaces can be recovered as special cases of the present analysis. The basic thermodynamic properties of the system under consideration, such as H elmholtz free energy, entropy, and surface excess, are derived. We show that using an equivalent sphere to approximate a spheroid can lead to an appreciable deviation in the prediction of the Helmholtz free energy. For a thin double layer, assuming a planar geometry will underestimate the Helmholtz free energy. (๑) 1997 Academic Press

Key Words: spheroidal charged surfaces, conductive and nonconductive; electrical potential distribution; arbitrary level; symmetric electrolyte solution; nonlinear Poisson- Boltzmann equation, perturbation solution, constant charges, constant potential; thermodynamic properties; Helmholtz free energy; entropy; surface excess.

\section{INTRODUCTION}

A system comprising a charged surface and an electrolyte solution is characterized by its spatial electrostatic potential distribution. The potential distribution, governed by the classic Poisson-Boltzmann equation (PBE), provides sufficient information for the estimation of the basic thermodynamic properties of the charged system. Although the solution to the general PBE is highly desirable for practical applications, it is almost impossible to derive, except under drastic assumptions. One of these is that the potential of the system is sufficiently low (the Debye-Huckel approximation). In this case, the PBE can be linearized and the resultant expres-

\footnotetext{
${ }^{1}$ To whom correspondence should be addressed.
}

sion solved analytically for some simple geometries (1). Gouy and Chapman were able to solve the PBE exactly for a planar surface of arbitrary potential immersed in a symmetric electrolyte solution (2). The analysis was extended by Hsu and Kuo (3) to asymmetric electrolytes through a semianalytical approach. For a nonplanar surface of arbitrary potential, the difficulty of solving PBE was circumvented either by adopting an approximate method (4-16) or by resorting to a numerical procedure (17-19). The former includes, for example, the results for cylindrical surfaces $(7,16,22,23)$ and spherical surfaces $(4-6,8-14,20-23)$.

The relevant analytical results reported in the literature for a charged surface in an electrolyte solution are almost always limited to simple geometries such as infinite flat plate, long cylinder, and sphere. In other words, an essentially onedimensional problem is considered. A model based on this consideration, although simplifying the mathematical treatment, is an approximation, at best, and can be unrealistic in practice. The linear size of a colloidal particle is finite, and various shapes can be assumed. For example, the kaolin particles used widely in coagulation experiments are finite and planar. Biological colloids are more complicated. They can be rod-like or olivary; even the shape of some cocci deviates appreciably from a sphere. Apparently, a more general geometry than the flat plate, cylinder, or sphere needs to be considered. The aim of the present work is to extend the classic analysis of the PBE to a two-dimensional space. One typical example is the spheroids, which simulate a wide class of dispersed entities in colloidal science. By varying the parameters that characterize the shape of a spheroid, different geometries can be portrayed (24). Previous efforts in the relevant studies were limited to the case of low surface potentials (25-27). In other words, a linearized PBE was solved. Here, a nonlinear PBE for a spheroidal surface is analyzed.

\section{ANALYSIS}

The electrostatic potential distribution for a charged surface immersed in an electrolyte solution is described by the 

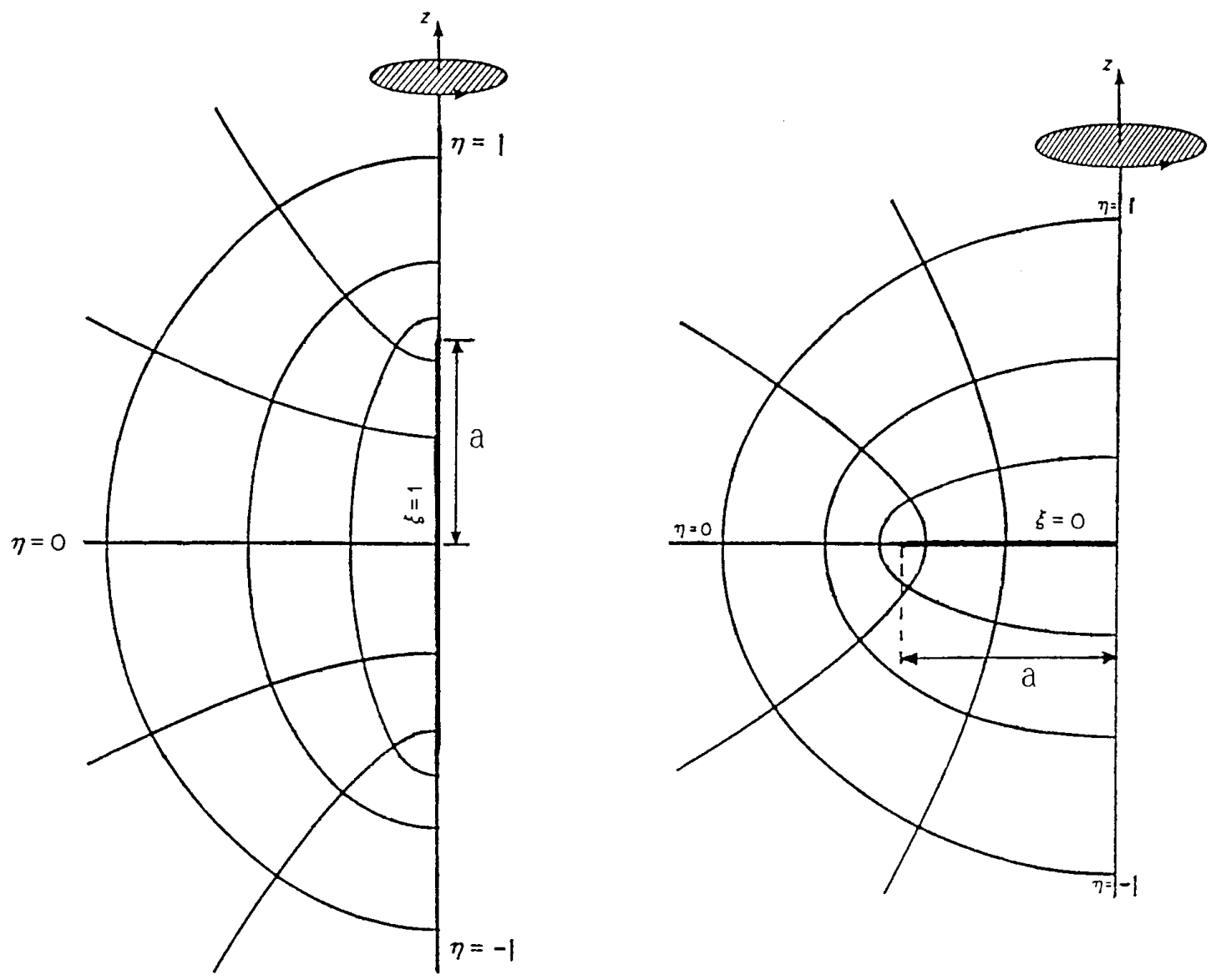

FIG. 1. Schematic representations of spheroids. (Left) prolate; (right) oblate.

Poisson-Boltzmann equation. For symmetric electrolytes this equation can be expressed as

$$
\nabla^{2} y=\kappa^{2} \sinh y
$$

where

$$
y=z e \psi / k_{\mathrm{B}} T
$$

and

$$
\kappa^{2}=8 \pi e^{2} I / \epsilon k_{\mathrm{B}} T
$$

In these expressions, $\nabla^{2}$ represents the Laplace operator, $\psi$ is the electrostatic potential, $\epsilon$ and $z$ denote, respectively, the dielectric constant and the valence of the electrolyte, $\kappa$ and $k_{\mathrm{B}}$ are, respectively, the reciprocal Debye length and the Boltzmann constant, $T$ denotes the absolute temperature, and $e$ and $I$ are, respectively, the elementary charge and the ionic strength.

Schematic representations of a prolate spheroid and an oblate spheroid are shown in Fig. 1 (left and right, respec- tively). Let $\xi$ and $\eta$ be, respectively, the radial and the angular coordinates, and let $a$ be the distance between the focus and the center of a spheroid. Equation [1] becomes

$$
\begin{aligned}
\frac{\partial}{\partial \eta}\left[\left(1-\eta^{2}\right) \frac{\partial y}{\partial \eta}\right]+\frac{\partial}{\partial \xi} & {\left[\left(\xi^{2}-j\right) \frac{\partial y}{\partial \xi}\right] } \\
& -c^{2}\left(\xi^{2}-j \eta^{2}\right) \sinh y=0
\end{aligned}
$$

where $j$ is an index ( $j=1$ for prolate spheroid, $j=-1$ for oblate spheroid) and $c=\kappa a$. We define

$$
\begin{aligned}
& R_{\mathrm{s}}=\sqrt{\frac{\xi_{\mathrm{s}}^{2}-(1+j) / 2}{\xi_{\mathrm{s}}^{2}+(1-j) / 2}} \\
& \lambda=\frac{1}{c \sqrt{\xi_{\mathrm{s}}^{2}+(1-j) / 2}} .
\end{aligned}
$$

Here, $R_{\mathrm{s}}$ is the ratio (length of minor axis/length of major axis), $\lambda$ denotes the ratio (Debye length/length of semimajor axis), and $\xi=\xi_{\mathrm{s}}$ means the surface of a spheroid. Making the transformation 


$$
\xi=A \lambda X^{\prime}+\xi_{\mathrm{s}}
$$

with

$$
A=\sqrt{\xi_{\mathrm{s}}^{2}+(1-j) / 2}
$$

Eq. [2] becomes

$$
\begin{aligned}
\lambda^{2} A^{2} \frac{\partial}{\partial \eta}[(1 & \left.\left.-\eta^{2}\right) \frac{\partial y}{\partial \eta}\right]+\frac{\partial}{\partial X^{\prime}} \\
\times & {\left.\left[\left(A \lambda X^{\prime}+\xi_{\mathrm{s}}\right)^{2}-j\right) \frac{\partial y}{\partial X^{\prime}}\right] } \\
& -\left[\left(A \lambda X^{\prime}+\xi_{\mathrm{s}}\right)^{2}-j \eta^{2}\right] \sinh y=0 .
\end{aligned}
$$

If a double layer is thin compared with the length of the semimajor axis, $y$ can be expanded as

$$
y=y_{0}+\lambda y_{1}+\lambda^{2} y_{2}+\cdots
$$

Substituting this expression into Eq. [5], and collecting terms of the same order of $\lambda$, we obtain

$$
\frac{\partial^{2} y_{0}}{\partial X^{\prime 2}}-h_{\mathrm{s}}^{2} \sinh y_{0}=0
$$

$\frac{\partial^{2} y_{1}}{\partial X^{\prime 2}}-h_{\mathrm{s}}^{2} y_{1} \cosh y_{0}=2 A f X^{\prime} \sinh y_{0}$

$$
-2 A f \frac{\partial y_{0}}{\partial X^{\prime}}-2 A f X^{\prime} \frac{\partial^{2} y_{0}}{\partial X^{\prime 2}}
$$

$$
\begin{aligned}
\frac{\partial^{2} y_{n}}{\partial X^{\prime 2}}-h_{\mathrm{s}}^{2} y_{n}^{\prime}= & 2 A f X^{\prime} y_{n-1}^{\prime}-2 A f \frac{\partial y_{n-1}}{\partial X^{\prime}} \\
& -2 A f X^{\prime} \frac{\partial^{2} y_{n-1}}{\partial X^{\prime 2}}+\frac{A^{2} f}{\xi_{\mathrm{s}}} X^{\prime 2} y_{n-2}^{\prime} \\
& -\frac{2 A^{2} f}{\xi_{\mathrm{s}}} X^{\prime} \frac{\partial y_{n-2}}{\partial X^{\prime}}-\frac{A^{2} f}{\xi_{\mathrm{s}}} X^{\prime 2} \frac{\partial^{2} y_{n-2}}{\partial X^{\prime 2}} \\
& -\frac{A^{2} f}{\xi_{\mathrm{s}}} \frac{\partial}{\partial \eta}\left[\left(1-\eta^{2}\right) \frac{\partial y_{n-2}}{\partial \eta}\right], n \geqslant 2
\end{aligned}
$$

where

$$
\begin{aligned}
f & =\left(\xi_{\mathrm{s}}^{2}-j\right) / \xi_{\mathrm{s}}^{2} \\
y_{n}^{\prime} & =\left(\frac{\partial^{n} \sinh y}{\partial \lambda^{n}}\right)_{\epsilon=0} / n ! \\
h_{\mathrm{s}} & =\sqrt{\frac{\xi_{\mathrm{s}}^{2}-j \eta^{2}}{\xi_{\mathrm{s}}^{2}-j}} .
\end{aligned}
$$

The boundary conditions at the surface associated with Eq. [1] depend on its nature. Here, we consider two types of surface: constant potential and constant amount of charge.

\subsection{Constant Surface Potential}

Let $\psi_{\mathrm{s}}$ be the potential at the surface of a spheroid $(\xi=$ $\xi_{\mathrm{s}}$ ), and let $y_{\mathrm{s}}$ be the corresponding value of $y$. The boundary conditions associated with Eqs. [7a]-[7c] are

$$
\begin{array}{ll}
y_{0}=y_{\mathrm{s}}, \text { and } y_{n}=0, & n \geqslant 1 \text { at } \xi=\xi_{\mathrm{s}} \\
y_{n} \rightarrow 0, & n \geqslant 0 \text { as } \xi \rightarrow \infty \\
\partial y_{n} / \partial \eta=0, & n \geqslant 0 \text { at } \eta=0 .
\end{array}
$$

The solution to Eq. [7a] subject to Eqs. [8a]-[8c] is

$$
y_{0}=2 \ln \left[\frac{1+\alpha \exp \left(-h_{\mathrm{s}} X^{\prime}\right)}{1-\alpha \exp \left(-h_{\mathrm{s}} X^{\prime}\right)}\right] \text {, }
$$

where

$$
\alpha=\tanh \left(y_{\mathrm{s}} / 4\right)
$$

Note that, by referring to Eq. [7f], $h_{\mathrm{s}}$ is the dimensionless metric coefficient at $\xi=\xi_{\mathrm{s}}$. Therefore the term $h_{\mathrm{s}} X^{\prime}$ on the right-hand side of Eq. [9] represents the dimensionless distance. Thus, Eq. [9] is equivalent to the result of Gouy and Chapman for a planar surface (2).

For convenience, we let $X=h_{\mathrm{s}} X^{\prime}$. Substituting Eq. [9] into Eq. [7b], assuming $y_{1}=g \cdot \sinh \left(y_{0} / 2\right)$, and solving the resultant expression subject to Eqs. [8a]-[8c], we obtain

$$
\begin{aligned}
y_{1}= & A_{1} \cdot\left[-\operatorname{csch}\left(\frac{y_{0}}{2}\right)+\frac{3 \cdot \ln (\alpha)}{2} \sinh \left(\frac{y_{0}}{2}\right)\right. \\
& \times \ln \left(\tanh \left(\frac{y_{0}}{4}\right)\right)-\frac{\ln (\alpha)}{2} \operatorname{coth}\left(\frac{y_{0}}{2}\right) \\
& \left.-\sinh \left(\frac{y_{0}}{2}\right) \ln ^{2}\left(\tanh \left(\frac{y_{0}}{4}\right)\right)\right]-2 \cdot A_{2} \\
& \times \operatorname{csch}\left(\frac{y_{0}}{2}\right)+\frac{C_{1}}{2}\left[\operatorname{coth}\left(\frac{y_{0}}{2}\right)+\sinh \left(\frac{y_{0}}{2}\right)\right. \\
& \left.\times \ln \left(\tanh \left(\frac{y_{0}}{4}\right)\right)\right]+C_{2} \cdot \sinh \left(\frac{y_{0}}{2}\right),
\end{aligned}
$$


where

$$
\begin{aligned}
A_{1}= & \frac{A \cdot f}{h_{\mathrm{s}}}\left(\frac{1}{h_{\mathrm{s}}^{2}}-1\right) \\
A_{2}= & A \cdot f / h_{\mathrm{s}} \\
C_{1}= & A_{1} \cdot(2+\ln (\alpha))+4 \cdot A_{2} \\
C_{2}= & \left(A_{1}+2 \cdot A_{2}\right) \cdot\left(1-\cosh \left(\frac{y_{\mathrm{s}}}{2}\right)\right) \operatorname{csch}^{2}\left(\frac{y_{\mathrm{s}}}{2}\right) \\
& -\ln (\alpha) \cdot\left(A_{1} \cdot \ln (\alpha)+A_{1}+2 \cdot A_{2}\right)
\end{aligned}
$$

In general, solving Eq. [7c] for higher order solutions, $y_{n}$, $n \geqslant 2$, is nontrivial; some general discussions on this are given in Appendix. However, if $y_{\mathrm{s}}$ is small, it can be shown that the solution to Eqs. [7a]-[7c] subject to Eqs. [8a]$[8 \mathrm{c}]$ is

$$
y_{n}=y_{\mathrm{s}}\left[\sum_{i=0}^{2 n} D_{n, i} X^{i}\right] \exp (-X),
$$

where

$$
\begin{aligned}
D_{0,0}= & 1 \\
D_{i, 0}= & 0, i=1,2,3 \cdots \\
D_{1,1}= & -\left(A_{1}+2 \cdot A_{2}\right) / 2 \\
D_{1,1}= & -A_{1} / 2 \\
D_{2,1}= & \frac{5 A^{2} f^{2}}{8 h_{\mathrm{s}}^{6}}-\frac{A^{2} f^{2}}{4 h_{\mathrm{s}}^{4}}+\frac{A^{2} f^{2}}{8 h_{\mathrm{s}}^{2}} \\
& +\frac{j \eta^{2} A^{2} f^{2}}{2 h_{\mathrm{s}}^{4} \xi_{\mathrm{s}}^{2}}-\frac{A^{2} f}{4 h_{\mathrm{s}}^{4} \xi_{\mathrm{s}}}-\frac{A^{2} f}{4 h_{\mathrm{s}}^{2} \xi_{\mathrm{s}}} \cdots .
\end{aligned}
$$

\subsection{Constant Amount of Surface Charge:}

\section{Nonconductive Surface}

Suppose that a certain amount of fixed charges are distributed uniformly over a nonconductive surface. A typical example for this type of surface is the inorganic colloids where the fixed charges on a surface arise from an irreversible adsorption of ions from the liquid phase. Since the dielectric constant of liquid phase is usually much greater than that of solid phase, the boundary condition at the charged surface can be written as

$$
\mathbf{n} \cdot \nabla \mathrm{y}=-\frac{4 \pi z e \sigma}{\epsilon k_{\mathrm{B}} T} \text { at } \xi=\xi_{\mathrm{s}},
$$

where $\mathbf{n}$ denotes the unit outer-normal vector of the surface and $\nabla$ and $\sigma$ are, respectively, the gradient operator and the surface charge density.

The solution to the zeroth-order perturbation equation, Eq. [7a], subject to Eq. [12], is Eq. [9] with

$$
\alpha=\tanh \left[\frac{\sinh ^{-1}\left(\sigma^{\prime} / 2\right)}{2}\right] \text {, }
$$

where

$$
\sigma^{\prime}=\frac{4 \pi \cdot a \cdot z \cdot \mathrm{e} \cdot \sigma}{\epsilon \cdot c \cdot k_{\mathrm{B}} \cdot T}
$$

The solution to the first-order perturbation equation, Eq. [7b], is Eq. [10] with Eqs. [10a]-[10c], and $C_{2}$ is replaced by

$$
\begin{aligned}
A_{1} \cdot\left[\frac{\operatorname{sech}\left(y_{\mathrm{s} 0}\right) \cdot \ln (\alpha)}{2}\right. & \left.-\ln ^{2}(\alpha) / 2\right] \\
& -C_{1} \cdot\left[\ln (\alpha)+\operatorname{sech}\left(y_{\mathrm{s} 0} / 2\right)\right] / 2
\end{aligned}
$$

with

$$
y_{\mathrm{s} 0}=2 \cdot \sinh ^{-1}\left(\frac{\sigma^{\prime}}{2}\right) .
$$

If $y_{\mathrm{s}}$ is small, the solution to the system of equations, Eqs. $[7 \mathrm{a}]-[7 \mathrm{c}]$, is

$$
y_{n}=\sigma^{\prime}\left[\sum_{i=0}^{2 n} D_{n, i} X^{i}\right] \exp (-X),
$$

where

$$
\begin{aligned}
D_{0,0}= & 1 \\
D_{1,0}= & D_{1.1}=-\left(A_{1}-2 \cdot A_{2}\right) / 2 \\
D_{1,2}= & -A_{1} / 2 \\
D_{2,0}= & D_{2,1} \\
= & \frac{7 A^{2} f^{2}}{8 h_{\mathrm{s}}^{6}}+\frac{A^{2} f^{2}}{4 h_{\mathrm{s}}^{4}}+\frac{3 A^{2} f^{2}}{8 h_{\mathrm{s}}^{2}}+\frac{j \eta^{2} A^{2} f^{2}}{2 h_{\mathrm{s}}^{4} \xi_{\mathrm{s}}^{2}} \\
& -\frac{A^{2} f}{4 h_{\mathrm{s}}^{4} \xi_{\mathrm{s}}}-\frac{A^{2} f}{4 h_{\mathrm{s}}^{2} \xi_{\mathrm{s}}} \cdots
\end{aligned}
$$

\subsection{Constant Amount of Surface Charge: \\ Conductive Surface}

Let us consider the case a certain amount of fixed charges are distributed over a conductive surface. Since charges are allowed to migrate over the surface, an equilibrium distribu- 


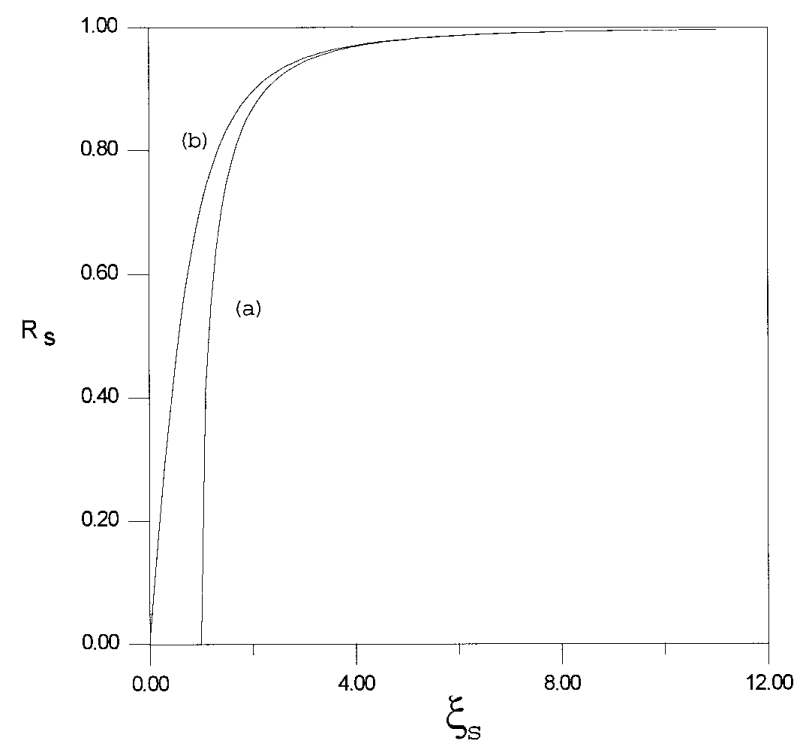

FIG. 2. Variation in the ratio (length of minor axis/length of major axis) as a function of $\xi_{\mathrm{s}}$. (a) prolate, (b) oblate.

tion will achieve. Surfaces bearing ionizable functional groups which are capable of undergoing reversible dissociation reactions in an electrolyte solution belong to this type of problem. Here, the boundary condition at the surface can be written as

$$
\left(\frac{\partial y}{\partial X}\right)_{X=0}=-\sigma^{\prime}(\eta)
$$

Since the potential is the same at each point on the charged surface, the solution of Eq. [1] can be obtained by following the procedure described in Section 2.1 with boundary condition at surface described by Eq. [16]. Apparently, the function $\sigma(\eta)$ needs to be determined. Let $q$ be the total amount of charges on solid surface. Integrating Eq. [16] yields

$$
\int_{0}^{1}\left(\frac{\partial y}{\partial X}\right)_{X=0} \cdot h_{\mathrm{s}} \cdot d \eta=\frac{-4 \pi z e q}{a\left(\xi_{\mathrm{s}}^{2}-j\right) \epsilon c k_{\mathrm{B}} T}=-q^{\prime} .
$$

For a given $q^{\prime}$, this expression can be used to evaluate $y_{\mathrm{s}}$. Substituting the value of $y_{\mathrm{s}}$ obtained into Eq. [16] yields the relation $\sigma(\eta)$. Since Eq. [16] leads to a nonlinear equation in $y_{\mathrm{s}}$, a numerical scheme is necessary, in general. However, if the potential is low, an analytical expression for $y_{\mathrm{s}}$ can be obtained. In this case, solving Eq. [17] for $y_{\mathrm{s}}$ gives

$$
y_{\mathrm{s}}=\frac{q^{\prime}}{\int_{0}^{1}\left(1+\lambda \cdot D_{1,1}+\lambda^{2} \cdot D_{2,1}+\cdots\right) h_{\mathrm{s}} \cdot d \eta} .
$$

Substituting this expression into Eq. [16], we obtain

$$
\sigma^{\prime}=y_{\mathrm{s}}\left(1+\lambda \cdot D_{1,1}+\lambda^{2} \cdot D_{2,1}+\cdots\right) .
$$

\section{DISCUSSION}

The simulated variation in the ratio $R_{\mathrm{s}}$ (length of minor axis/length of major axis) as a function of $\xi_{\mathrm{s}}$ is shown in Fig. 2. As can be seen from this figure, if $\xi_{\mathrm{s}}>10, R_{\mathrm{s}}$ is essentially unity; i.e., a spheroid can be approximated by a sphere. If $\xi_{\mathrm{s}}=(j+1) / 2, R_{\mathrm{s}}$ vanishes. In this case a prolate spheroid becomes a needle-like object, and an oblate reduces to a disk. Figure 3 shows that the variation in the ratio $\lambda$ (Debye length/length of semi-major axis) as a function of the parameter $c$ at various values of $\xi_{\mathrm{s}}$. This figure reveals that, the greater the value of $c$, the smaller the relative thickness of double layer. For a fixed value of $c, \lambda$ decreases with the increase in $\xi_{\mathrm{s}}$.

Figure 4 shows the variation in the dimensionless potential as a function of position variable $\left(\xi-\xi_{\mathrm{s}}\right)$ at various values of $\eta$ for a prolate. As can be seen from this figure, for a fixed $\left(\xi-\xi_{\mathrm{s}}\right)$, the dimensionless potential at $\eta=1$ is higher than that at $\eta=0$. This means that potential increases with curvature. By referring to Fig. 2, the greater the $\xi_{\mathrm{s}}$, the closer a prolate to a sphere, and the closer the two curves shown in Fig. 4. On the other hand, the smaller the $\xi_{s}$, the closer a prolate to a needle-like object, and the more distinct the two curves. Note that the distance between two equal-potential surfaces in the insert of Fig. 4 is not equal for different values of $\eta$, e.g., the distance at $\eta=0$ is greater than that at $\eta=1$.

Figure 5 shows the variation in the surface properties as a function of the position variable $\eta$ for both nonconductive and conductive prolate spheroidal surfaces at a constant amount of surface charges. As can be seen from Fig. 5, for

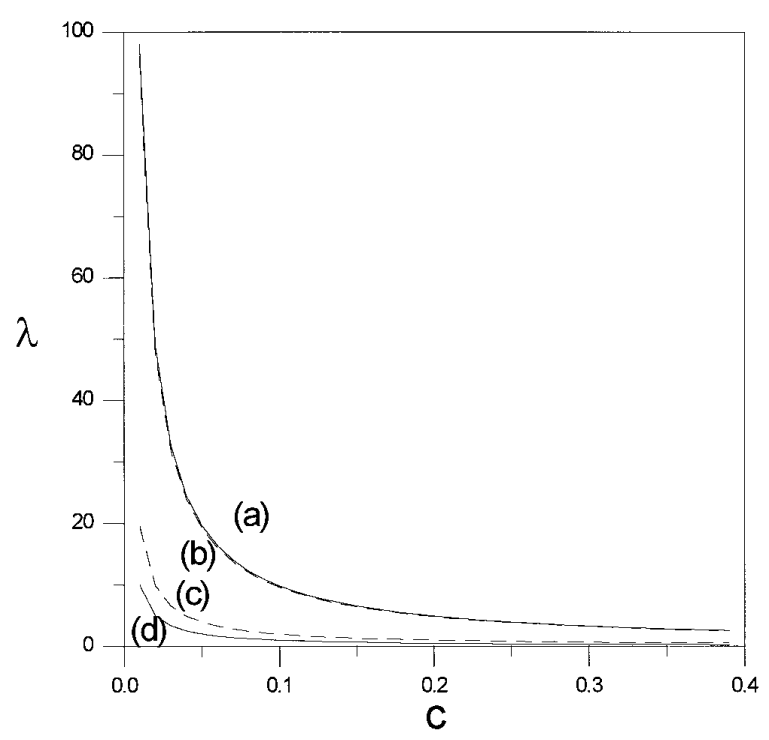

FIG . 3. Variation in the ratio (Debye length/length of semimajor axis) as a function of $c$. Solid line, prolate; dashed line, oblate. (a) $\xi_{\mathrm{s}}=1.02$; (b) $\xi_{\mathrm{s}}=0.3$; (c) $\xi_{\mathrm{s}}=5$; (d) $\xi_{\mathrm{s}}=10$. 
the case of a nonconductive surface the potential on the surface is position dependent; the greater the curvature, the lower the potential. Figure 5 also suggests that for a conductive surface the surface charge density increases with the curvature, as expected. The qualitative behavior of an oblate is similar to that of a prolate.

As $\xi_{\mathrm{s}} \rightarrow \infty$, Eq. [2] reduces to

$$
\frac{\partial^{2} y}{\partial \xi^{2}}+\frac{2}{\xi} \frac{\partial y}{\partial \xi}=c^{2} \sinh y .
$$

This is the Poisson-Boltzmann equation for spherical geometry and symmetric electrolytes. Also, Eq. [5] and Eq. [3b] become, respectively,

$$
\frac{\partial}{\partial X^{\prime}}\left[\left(1+\lambda X^{\prime}\right)^{2} \frac{\partial y}{\partial X^{\prime}}\right]-\left(1+\lambda X^{\prime}\right)^{2} \sinh y=0
$$

and

$$
\lambda=1 /\left(c \cdot \xi_{\mathrm{s}}\right) .
$$

On the basis of these expressions, a perturbation analysis can be conducted. This approach was adopted for the case of a spherical surface in a symmetric electrolyte solution (28).

It should be pointed out that the present perturbation method is not limited to a very thin double layer. Its applicability is related closely to $R_{\mathrm{s}}$, the ratio (length of semiminor axis/length of semimajor axis ). The greater the $R_{\mathrm{s}}$, the thicker a double layer can be assumed. For

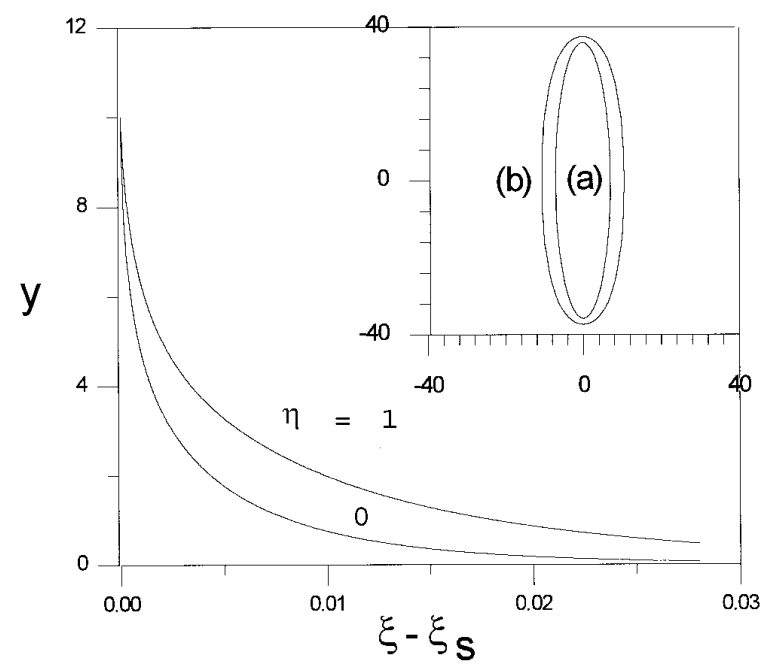

FIG. 4. Variation in the dimensionless potential $y$ as a function of position variable $\left(\xi-\xi_{\mathrm{s}}\right)$ for a prolate with $y_{\mathrm{s}}=10, \xi_{\mathrm{s}}=1.02\left(R_{\mathrm{s}}=\right.$ 0.197 ), and $\lambda=0.028$ at $\eta=0$ and $\eta=1$. Insert: projection of equalpotential surfaces on the $x-z$ plane. (a) $y=y_{\mathrm{s}}$; (b) $y=0.01 y_{\mathrm{s}}$.

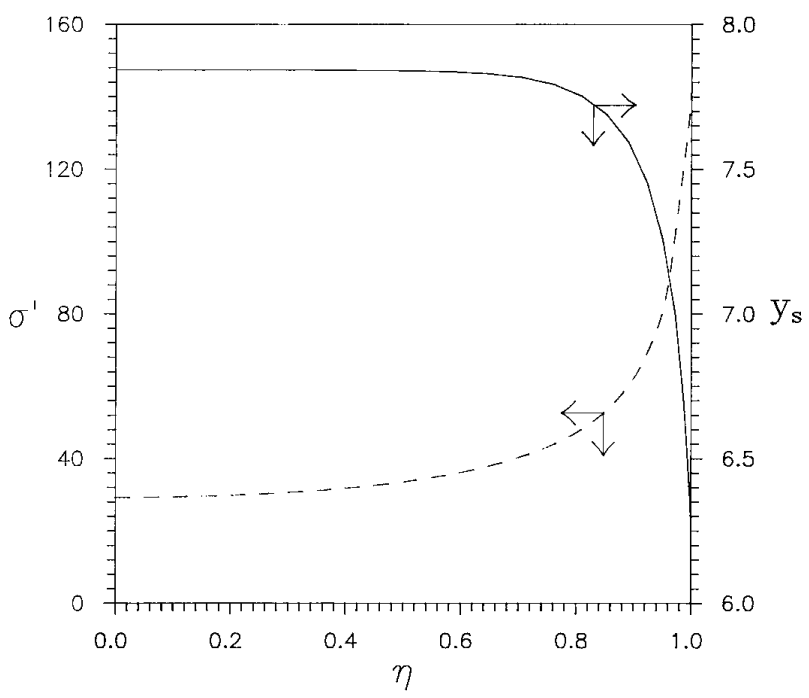

FIG. 5. Variation in the surface properties of a prolate with $\xi_{\mathrm{s}}=1.02$ $\left(R_{\mathrm{s}}=0.197\right)$ and $\lambda=0.028$ as a function of $\eta$. Solid line: nonconductive surface at constant amount of surface charges with $\sigma^{\prime}=100$; dashed line: conductive surface with $y_{\mathrm{s}}=10$.

instance, at $y_{\mathrm{s}}=2$ if $R_{\mathrm{s}}=0.2, \lambda$ is on the order of 0.03 , and if $R_{\mathrm{s}}=0.99, \lambda$ is on the order of 0.25 . Tables 1 and 2 illustrate a comparison between the first-order perturbation solution for potential distribution and the result obtained by assuming a planar geometry, i.e., the GouyChapman model (2). These tables reveal that the performance of the former is better than that of the latter. In other words, although a double layer is thin, assuming a planar geometry may still lead to an appreciable deviation. Note that, if a double layer is thin, only the local curvature of a charged surface is significant. In this case, an arbitrary surface can be approximated by the union of a set of spheroidal surfaces, each can be analyzed by the present method. This choice for the estimation of the electrical potential distribution is more preferred than using a planar surface. Tables 1 and 2 also show that, the percent deviation increases with the curvature of a surface (measured by $|\eta|$ ) and decreases with the increase in surface potential. For a fixed $\lambda$, the larger the $\xi_{\mathrm{s}}$, the smaller the percent deviation.

For the present system the dimensionless free energy, $F$, for the case of constant surface potential can be calculated by (2)

$$
F=-\int_{0}^{y_{\mathrm{s}}} \sigma^{\prime}(y) d y
$$

where $F=4 \pi a z^{2} e^{2} f / \lambda c k_{\mathrm{B}}^{2} T^{2}$ and $f$ is the free energy per unit area. Substituting Eq. [6] into Eq. [16] gives 


$$
\begin{aligned}
\sigma^{\prime}= & 2 \cdot \sinh \left(\frac{y_{\mathrm{s}}}{2}\right)-2 \lambda\left(A_{1}+2 \cdot A_{2}\right) \\
& \times \operatorname{csch}\left(\frac{y_{\mathrm{s}}}{2}\right)\left(1-\cosh \left(\frac{y_{\mathrm{s}}}{2}\right)\right)+\cdots
\end{aligned}
$$

Substituting Eq. [23] into Eq. [22] yields

$$
\begin{aligned}
F= & -4\left(\cosh \left(\frac{y_{\mathrm{s}}}{2}\right)-1\right) \\
& +4 \lambda\left(A_{1}+2 \cdot A_{2}\right) \cdot \ln \left(\operatorname{sech}^{2}\left(\frac{y_{\mathrm{s}}}{4}\right)\right)+\cdots
\end{aligned}
$$

The entropy of the electrical double layer can be calculated by

$$
\begin{aligned}
S & =-\left(\frac{\partial F}{\partial T}\right)_{V, n_{i}} \\
& =-2 \lambda\left(A_{1}+A_{2}\right) / T \cdot \ln \left(\operatorname{sech}^{2}\left(\frac{y_{\mathrm{s}}}{4}\right)\right)+\cdots
\end{aligned}
$$

The electroneutrality implies that

$$
\Gamma_{+}-\Gamma_{-}+\frac{\sigma}{z F}=0
$$

where $\Gamma_{+}$and $\Gamma_{-}$are the amounts of adsorbed cation and anion, respectively. The dimensionless surface excess of cation, $\Gamma$ * , can be evaluated by

$$
\begin{aligned}
\Gamma_{+}^{*}= & \frac{c \Gamma_{+}}{a C_{i \infty}}=\int_{0}^{\infty}[\exp (y)-1] d X=-2 \exp \left(\frac{y_{0}}{2}\right) \\
& -\frac{\epsilon}{2}\left\{\left(5 \cdot A_{1} \cdot \ln (\alpha)+8 \cdot A_{2}-C_{1}\right) \exp \left(\frac{y_{0}}{2}\right)\right. \\
& -C_{2} \cdot \exp \left(y_{0}\right)+\frac{4 \cdot A_{2}}{1-\exp \left(y_{0 / 2}\right)}-\frac{4\left(A_{1}+A_{2}\right)}{1+\exp (y / 2)} \\
& +\frac{-10 \cdot A_{1}-4 \cdot \ln (\alpha) \cdot A_{1}+4 \cdot A_{2}}{2} \\
& \times \ln \left[-1+\exp \left(y_{0} / 2\right)\right]+\frac{\exp \left(y_{0}\right)}{2} \\
& \times\left[8 \cdot A_{1}+3 \cdot A_{1} \cdot \ln (\alpha) \exp \left(\frac{y_{0}}{2}\right)\right. \\
& \left.\left.+C_{1} \cdot \exp \left(\frac{y_{0}}{2}\right)\right] \ln \left(\tanh \left(\frac{y_{0}}{4}\right)\right)\right\}+\cdots
\end{aligned}
$$

Figure 6 displays the simulated variation of $-F$ as a function of $\eta$. This figure reveals that the dimensionless Helmholtz free energy is sensitive to the variation of $\eta$ for $\eta>$

\begin{tabular}{|c|c|c|c|c|c|c|c|}
\hline \multirow[b]{2}{*}{$\xi_{\mathrm{s}}$} & \multirow[b]{2}{*}{$\lambda$} & \multicolumn{3}{|c|}{$E_{\mathrm{pt}}(\%)$} & \multicolumn{3}{|c|}{$E_{\mathrm{pl}}(\%)$} \\
\hline & & $\eta=0$ & $\eta=1$ & Average & $\eta=0$ & $\eta=1$ & Average \\
\hline 1.02 & 0.02 & 2.2 & 18.7 & 10.5 & 5.1 & 27.8 & 16.4 \\
\hline \multirow[t]{3}{*}{1.25} & 0.02 & 1.2 & 0.6 & 0.9 & 2.5 & 5.1 & 3.8 \\
\hline & 0.05 & 1.1 & 3.2 & 2.2 & 5.3 & 12 & 8.7 \\
\hline & 0.1 & 2.1 & 11.3 & 6.7 & 10.3 & 21.7 & 16 \\
\hline \multirow[t]{3}{*}{10} & 0.05 & 0.6 & 0.6 & 0.6 & 4.5 & 4.6 & 4.6 \\
\hline & 0.1 & 1.8 & 1.9 & 1.8 & 8.5 & 8.5 & 8.5 \\
\hline & 0.2 & 5.8 & 6.1 & 5.9 & 17 & 16.9 & 16.9 \\
\hline
\end{tabular}
0.8 . The diameter of most bacteria ranges from 0.2 to $2 \mu \mathrm{m}$ in diameter, and the length from 2 to $8 \mu \mathrm{m}$ (29). For surfaces of these linear sizes, Fig. 6 indicates that assuming a planar geometry will underestimate the Helmholtz free energy. Often, an irregular object is approximated by an equivalent sphere, the maximal linear size of the former is equal to the diameter of the latter. Let us consider the case where a prolate is approximated by an equivalent sphere, both having the same surface potential. Figure 7 shows the variation in the difference between the Helmholtz free energies of these

TABLE 1

Comparison between the First-Order Perturbation Solution for Potential Distribution (FPS) and the Result Obtained by Assuming a Planar Geometry, i.e., Gouy-Chapman Model (GCM), for Various $\xi_{\mathrm{s}}$ and $\lambda$ for the Case $y_{\mathrm{s}}=1$

Note. $E_{\mathrm{pt}}$ : percent deviation of FPS; $E_{\mathrm{pl}}$ : percent deviation of GCM. The percent deviation, $E$, is defined as

$$
E=100 \% \cdot \int_{0}^{\infty}\left|y_{\text {exact }}-y_{\text {approximate }}\right| d X / \int_{0}^{\infty} y_{\text {exact }} d X .
$$


TABLE 2

Comparison between the First-Order Perturbation Solution for Potential Distribution (FPS) and the Result Obtained by Assuming a Planar G eometry, i.e., G ouy-Chapman Model (GCM), for Various $\xi_{s}$ and $\lambda$ for the Case of Table 1, except that $y_{s}=4$

\begin{tabular}{|c|c|c|c|c|c|c|c|}
\hline \multirow[b]{2}{*}{$\xi_{\mathrm{s}}$} & \multirow[b]{2}{*}{$\lambda$} & \multicolumn{3}{|c|}{$E_{\mathrm{pt}}(\%)$} & \multicolumn{3}{|c|}{$E_{\mathrm{pl}}(\%)$} \\
\hline & & $\eta=0$ & $\eta=1$ & Average & $\eta=0$ & $\eta=1$ & Average \\
\hline 1.02 & 0.02 & 5.4 & 12.7 & 9 & 7.7 & 22.1 & 14.9 \\
\hline \multirow[t]{3}{*}{1.25} & 0.02 & 0.9 & 0.6 & 0.7 & 2.2 & 4.2 & 3.2 \\
\hline & 0.05 & 0.9 & 2.9 & 1.9 & 4.5 & 9.1 & 6.8 \\
\hline & 0.1 & 1.7 & 8.7 & 5.2 & 8.5 & 16.99 & 12.7 \\
\hline \multirow[t]{3}{*}{10} & 0.05 & 0.5 & 0.5 & 0.5 & 3.8 & 3.8 & 3.8 \\
\hline & 0.1 & 1.3 & 1.4 & 1.4 & 6.8 & 6.8 & 6.8 \\
\hline & 0.2 & 4.6 & 4.9 & 4.8 & 13.1 & 13.2 & 13.1 \\
\hline
\end{tabular}

two objects as a function of $R_{\mathrm{s}}$. As can be seen from this figure, assuming an equivalent sphere can lead to an appreciable deviation if $R_{\mathrm{s}}$ is smaller than 0.4 , i.e., the minor axis is $4 / 10$ the major axis, even at a low surface potential.

Solving directly the two-dimensional, nonlinear partial differential equation in curvilinear coordinates, Eq. [2], through a numerical method can be nontrivial. In a study of the double-layer interaction between two parallel cylinders, Lyne et al. (30) proposed an iterative procedure for the resolution of a nonlinear PBE. This approach, although may be extended to spheroidal coordinates, requires some underrelaxation of iterated estimates for relatively high surface potentials. In general, solving Eq. [2] numerically requires considerable computing efforts, even for the limiting case of spherical surfaces (31). In contrast, the present semianalytical approach provides an efficient way for determining

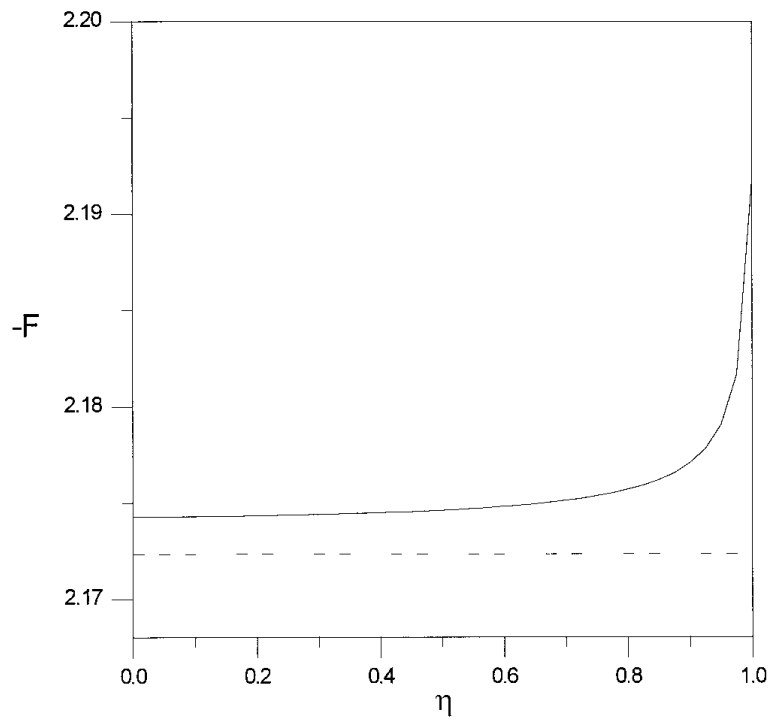

FIG. 6. Variation in the negative dimensionless Helmholtz free energy as a function of $\eta$. Solid line represents the exact value, and dashed line denotes the result for a planar surface. Parameters: major axis $=2 \mu \mathrm{m}$, minor axis $=0.4 \mu \mathrm{m}, z=1, I=0.001 \mathrm{~mol} / \mathrm{m}^{3}, T=298.15^{\circ} \mathrm{K}, \epsilon=78$, and $y_{\mathrm{s}}=2$. the electrostatic potential distribution around spheroidal surfaces for thin to medium thick double layers. It is a complementary result to those for thick double layers $(25,27)$.

\section{CONCLUSION}

In summary, the electrical potential distribution for a spheroidal surface immersed in a symmetric electrolyte solution is analyzed, and the basic thermodynamic properties, such as Helmholtz free energy, entropy, and surface excess, are derived. A perturbation approach is adopted to solve the governing Poisson-Boltzmann equation. We show that using an equivalent sphere to approximate a spheroid may lead to an appreciable deviation in the prediction of the Helmholtz free energy even at a low surface potential. Also, assuming

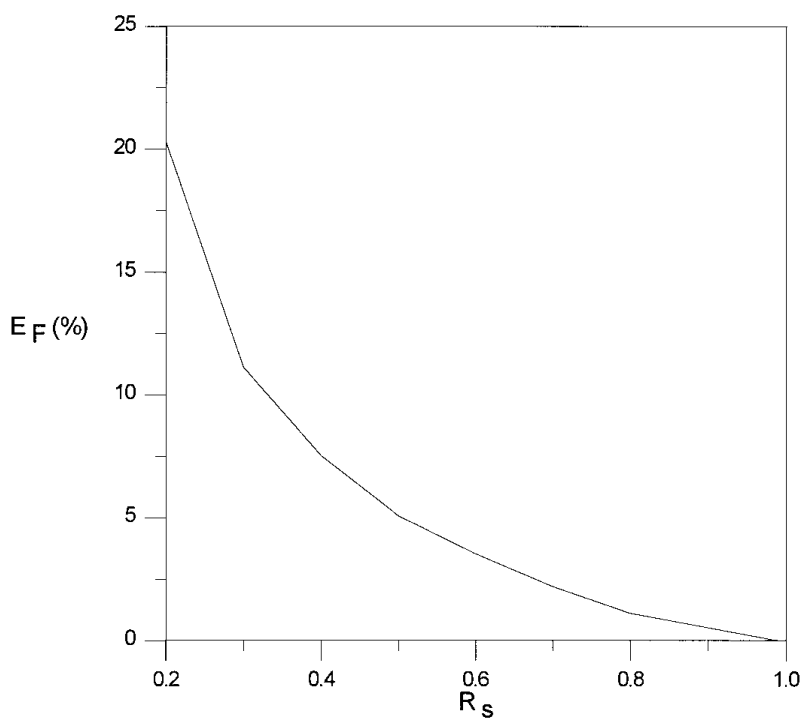

FIG. 7. Variation in the difference between the average Helmholtz free energy of a prolate, $F_{\mathrm{p}}$, and that of an equivalent sphere, $F_{\text {eq }}$, measured by $E_{\mathrm{F}}=100 \%\left|\left(F_{\mathrm{p}}-F_{\mathrm{eq}}\right) / F_{\mathrm{p}}\right|$, as a function of $R_{\mathrm{s}}$. The maximal linear size of the prolate is equal to the diameter of the sphere, and both have the same surface potential, which is sufficiently low, and $\lambda=0.1$. 
a planar geometry for the case of thin electrical double layer can be inappropriate.

\section{APPENDIX}

Equation [7c] represents a set of linear, second-order, nomhomogeneous differential equations with its constituent homogeneous equation

$$
\frac{\partial^{2} y_{n}}{\partial X^{2}}-y_{n} \cdot \cosh \left(y_{0}\right)=0
$$

Let $y_{n, \mathrm{~h}}$ be the solution of this equation. Assume

$$
y_{n, \mathrm{~h}}=g_{n, \mathrm{~h}} \cdot \sinh \left(y_{0} / 2\right) .
$$

Substituting Eq. [A2] into Eq. [A1] yields

$$
\frac{\partial^{2} g_{n, \mathrm{~h}}}{\partial X^{2}}-2 \cosh \left(\frac{y_{0}}{2}\right) \cdot \frac{\partial g_{n, \mathrm{~h}}}{\partial X}=0 .
$$

Solving this equation, we obtain

$$
\begin{aligned}
g_{n, \mathrm{~h}}=E_{1} \cdot\left[\operatorname{coth}\left(\frac{y_{0}}{2}\right)\right. & \csc \mathrm{h}\left(\frac{y_{0}}{2}\right) \\
& \left.+\ln \left(\tanh \left(\frac{y_{0}}{4}\right)\right)\right]+E_{2}
\end{aligned}
$$

where $E_{1}$ and $E_{2}$ are arbitrary constants. Therefore

$$
\begin{array}{r}
y_{n, \mathrm{~h}}=E_{1} \cdot\left[\operatorname{coth}\left(\frac{y_{0}}{2}\right)+\sinh \left(\frac{y_{0}}{2}\right) \ln \left(\tanh \left(\frac{y_{0}}{4}\right)\right)\right] \\
+E_{2} \cdot \sinh \left(\frac{y_{0}}{2}\right) .
\end{array}
$$

Note that, although the solution of Eq. [7c] can be retrieved directly by a numerical scheme, considerable computational efforts are inevitable. Here, we suggest using Eq. [A5] to evaluate the complementary solution, $y_{n, \mathrm{~h}}$, and then solve Eq. [7c] for a particular solution. This will reduce the computational efforts considerably.

\section{ACKNOWLEDGMENT}

This work was supported by the National Science Council of the Republic of China under Grant NSC84-2214-E002-030.

\section{REFERENCES}

1. Hiemenz, P. C., in "Principles of Colloid and Surface Chemistry,", 2nd ed. Dekker, New York, 1986.

2. Hunter, R. J., in "Foundations of Colloid Science," Vol. 1. Oxford Univ. Press, London, 1989.

3. Hsu, J. P., and Kuo, Y. C., J. Chem. Soc. Faraday Trans. 89, 1229 (1993).

4. La Mer, V. K., Gronwall, T. H., and Greiff, L. J., J. Phys. Chem. 35, 2245 (1931).

5. Dukhin, S. S., Semenikhin, N. M., and Shapinskaya, L. M., Dokl. Phys. Chem. 193, 540 (1970).

6. Dukhin, S. S., Semenikhin, N. M., and Shapinskaya, L. M., Dokl. Akad. Nauk SSSR 193, 385 (1970).

7. Sigal, V. L., and Shamansky, V. E., Dopov. Akad. Nauk Ukr. RSR 4, 346 (1970).

8. Parlange, J. Y., J. Chem. Phys. 57, 376 (1972).

9. Abraham-Schrauner, B., J. Colloid Interface Sci. 44, 79 (1973).

10. Brenner, S. L., and Roberts, R. E., J. Phys. Chem. 77, 2367 (1973).

11. White, L. R., J. Chem. Soc. Faraday Trans. 2 73, 577 (1977).

12. Bentz, J., J. Colloid Interface Sci. 80, 179 (1981).

13. Ohshima, H., Healy, T. W., and White, L. R., J. Colloid Interface Sci. 90, 17 (1982).

14. Natarajan, R., and Schechter, R. S., J. Colloid Interface Sci. 99, 50 (1984).

15. Ohshima, H., and Ohki, S., Biophys. J. 47, 673 (1985).

16. Hsu, J. P., and Kuo, Y. C., J. Colloid Interface Sci. 167, 35 (1994).

17. Leob, A. L., Wiersema, P. H., and Overbeek, J. Th. G., in "The Electrical Double Layer Around a Spherical Colloidal Particle.'” MIT Press, Cambridge, MA, 1961.

18. Stigter, D., J. Electroanal. Chem. 37, 61 (1972).

19. Chang, C. F. R., and Sposito, G., J. Colloid Interface Sci. 163, 19 (1994).

20. Stokes, A. N., J. Chem. Phys. 65, 261 (1976).

21. Overbeek, J. Th. G., Verhoeckx, G. J., de Bruyn, P. L., and Lekkerkerker, H. N. W., J. Colloid Interface Sci. 119, 422 (1987).

22. Lekkerkerker, H. N. W., Physica A 159, 319 (1989).

23. van Aken, G. A., Lekkerkerker, H. N. W., Overbeek, J. Th. G., and de Bruyn, P. L., J. Phys. Chem. 94, 8468 (1990).

24. Moon, P., and Spencer, D. E., in 'FField Theory Handbook.' SpringerVerlag, Berlin, 1961.

25. Flammer, C., in "'Spheroidal Wave Functions." Stanford Univ. Press, Stanford, CA, 1957.

26. Yoon, B. Y., and Kim, S., J. Colloid Interface Sci. 128, 275 (1989).

27. Hsu, J. P., and Liu, B. T., J. Colloid Interface Sci. 175, 785 (1996).

28. Abraham-Shraunter, B., J. Colloid Interface Sci. 44, 79 (1973).

29. Tortora, G. J., Funke, B. R., and Case, C. L., in "Microbiology: An Introduction,', 4th ed. Redwood, CA, 1992.

30. Lyne, M. P., Bowen, B. D., and Levine, S., J. Colloid Interface Sci. 150, 374 (1992).

31. Carnie, S. L., Chan, D. Y. C., and Stankovich, J., J. Colloid Interface Sci. 165, 116 (1994). 